\title{
Sources, composition, and mixing state of submicron particulates over central Indo-
} Gangetic Plain

\author{
Nandita Singh ${ }^{1,2}$, Tirthankar Banerjee ${ }^{1,2,3 *}$, Karine Deboudt ${ }^{2}$, Abhishek Chakraborty $^{4}$, Md Firoz \\ Khan ${ }^{5}$ and Mohd Talib Latif 6 \\ ${ }^{1}$ Institute of Environment and Sustainable Development, Banaras Hindu University, Varanasi 742225, India \\ 'Laboratoire de Physico-Chimie de l'Atmosphère, Université du Littoral Côte d'Opale, Dunkerque 59140, France \\ ${ }^{3}$ DST-Mahamana Centre of Excellence in Climate Change Research, Banaras Hindu University, Varanasi 742225, India \\ ${ }^{4}$ Department of Environmental Science \& Engineering, Indian Institute of Technology- Bombay, Powai 400076, India \\ ${ }^{5}$ Department of Chemistry, Faculty of Science, University of Malaya, Kuala Lumpur 50603, Malaysia \\ ${ }^{6}$ Department of Earth Sciences and Environment, Universiti Kebangsaan Malaysia, Bangi 43600, Malaysia \\ *Correspondence: tb.iesd@bhu.ac.in; tirthankaronline@gmail.com
}

\section{List of Tables and Figures}

Table S1. Descriptive statistics of all identified chemical species in submicron particulates.

Table S2. Mass concentration and diagnostic ratio of organic species.

Table S3. List of Abbreviations.

Table S4. Neutralization factor (NF) for $\mathrm{NH}_{4}{ }^{+}, \mathrm{Ca}^{2+}, \mathrm{Mg}^{2+}$ against $\mathrm{SO}_{4}{ }^{2-}$ and $\mathrm{NO}_{3}{ }^{-}$.

Table S5. Chemical and physical characteristics of different types of submicron particles.

Table S6. List of selected elements, ions and molecular markers for source identification.

Fig. S1. Seasonal variation in relative contribution of various species in submicron particulates.

Fig. S2. Factor profile for various sources.

Fig. S3. Wind vectors over the study site. 
Table S1. Descriptive statistics of all identified chemical species in submicron particulates.

\begin{tabular}{|c|c|c|c|c|c|c|}
\hline Species & Mean & SD & Min & Max & $1^{\text {st }}$ Quartile & $3^{\text {rd }}$ Quartile \\
\hline $\mathrm{PM}_{1.1}$ & 69 & 55 & 11 & 274 & 28 & 106 \\
\hline $\mathrm{Na}^{+}$ & 1.5 & 1.3 & 0.1 & 6.3 & 0.4 & 2.1 \\
\hline $\mathrm{K}^{+}$ & 1.4 & 1.2 & 0.1 & 5.3 & 0.4 & 2.2 \\
\hline $\mathrm{NH}_{4}^{+}$ & 2.5 & 2.0 & 0.1 & 7.9 & 1.0 & 3.8 \\
\hline $\mathrm{Mg}^{2+}$ & 0.4 & 0.4 & 0.0 & 1.8 & 0.2 & 0.6 \\
\hline $\mathrm{Ca}^{2+}$ & 1.4 & 1.1 & 0.1 & 4.7 & 0.4 & 2.1 \\
\hline $\mathrm{Cl}^{-}$ & 1.4 & 1.3 & 0.0 & 6.3 & 0.4 & 2.1 \\
\hline $\mathrm{NO}_{3}^{-}$ & 4.4 & 4.0 & 0.1 & 15.5 & 1.1 & 7.4 \\
\hline $\mathrm{SO}_{4}{ }^{2-}$ & 6.6 & 5.0 & 0.4 & 18.7 & 2.8 & 10.9 \\
\hline $\mathrm{Ca}$ & 3.3 & 3.1 & 0.1 & 15.9 & 1.1 & 4.3 \\
\hline $\mathrm{Fe}$ & 0.6 & 1.0 & 0.1 & 5.4 & 0.1 & 0.6 \\
\hline K & 2.7 & 2.8 & 0.1 & 11.7 & 0.6 & 4.2 \\
\hline $\mathrm{Mg}$ & 1.1 & 1.0 & 0.0 & 5.2 & 0.4 & 1.5 \\
\hline $\mathrm{Na}$ & 2.7 & 2.0 & 0.2 & 8.4 & 1.2 & 3.7 \\
\hline $\mathrm{Zn}$ & 0.89 & 1.28 & 0.01 & 5.53 & 0.04 & 1.38 \\
\hline $\mathrm{Cr}$ & 0.040 & 0.024 & 0.001 & 0.114 & 0.019 & 0.057 \\
\hline $\mathrm{Mn}$ & 0.017 & 0.012 & 0.005 & 0.070 & 0.009 & 0.021 \\
\hline $\mathrm{Cu}$ & 0.007 & 0.011 & 0.001 & 0.095 & 0.003 & 0.007 \\
\hline $\mathrm{Pb}$ & 0.034 & 0.019 & 0.005 & 0.083 & 0.021 & 0.046 \\
\hline Co & 0.002 & 0.002 & 0.000 & 0.006 & 0.000 & 0.003 \\
\hline $\mathrm{Ni}$ & 0.004 & 0.003 & 0.000 & 0.017 & 0.002 & 0.006 \\
\hline $\mathrm{Cd}$ & 0.002 & 0.001 & 0.000 & 0.006 & 0.001 & 0.003 \\
\hline WSOC & 12.6 & 14.5 & 0.4 & 65.7 & 2.6 & 17.1 \\
\hline \multicolumn{7}{|l|}{ n-Alkanes } \\
\hline n-C17H36 & 59 & 33 & 8 & 138 & 34 & 79 \\
\hline n-C18H38 & 80 & 44 & 18 & 233 & 46 & 106 \\
\hline $\mathrm{n}-\mathrm{C} 19 \mathrm{H} 40$ & 69 & 43 & 6 & 203 & 30 & 97 \\
\hline $\mathrm{n}-\mathrm{C} 2 \mathrm{OH} 42$ & 89 & 65 & 4 & 299 & 33 & 141 \\
\hline $\mathrm{n}-\mathrm{C} 21 \mathrm{H} 44$ & 56 & 41 & 5 & 149 & 22 & 84 \\
\hline $\mathrm{n}-\mathrm{C} 22 \mathrm{H} 46$ & 32 & 23 & 3 & 103 & 16 & 45 \\
\hline $\mathrm{n}-\mathrm{C} 23 \mathrm{H} 48$ & 89 & 69 & 16 & 327 & 31 & 127 \\
\hline $\mathrm{n}-\mathrm{C} 24 \mathrm{H} 50$ & 28 & 18 & 6 & 114 & 13 & 37 \\
\hline n-C25H52 & 77 & 64 & 8 & 293 & 32 & 102 \\
\hline n-C26H54 & 35 & 23 & 5 & 110 & 19 & 42 \\
\hline n-C27H56 & 59 & 47 & 6 & 207 & 26 & 71 \\
\hline n-C28H58 & 57 & 42 & 8 & 197 & 25 & 83 \\
\hline $\mathrm{n}-\mathrm{C} 29 \mathrm{H} 60$ & 48 & 43 & 5 & 217 & 19 & 58 \\
\hline n-C3OH62 & 42 & 31 & 5 & 172 & 20 & 63 \\
\hline n-C31H64 & 61 & 43 & 3 & 217 & 31 & 85 \\
\hline n-C32H66 & 33 & 26 & 6 & 200 & 16 & 43 \\
\hline
\end{tabular}




$\begin{array}{lcccccc}\mathrm{n}-\mathrm{C} 33 \mathrm{H} 68 & 28 & 23 & 3 & 122 & 14 & 33 \\ \mathrm{n}-\mathrm{C} 34 \mathrm{H} 70 & 23 & 15 & 4 & 89 & 11 & 30 \\ \mathrm{n}-\mathrm{C} 35 \mathrm{H} 72 & 14 & 14 & 1 & 64 & 3 & 21 \\ \text { Subtotal } & \mathbf{9 7 9} & \mathbf{7 0 4} & \mathbf{1 1 9} & \mathbf{3 4 5 5} & \mathbf{4 4 1} & \mathbf{1 3 4 6}\end{array}$

Phthalates

$\begin{array}{lcccccc}\text { DEP } & 123 & 88 & 26 & 399 & 51 & 164 \\ \text { DBP } & 118 & 74 & 32 & 365 & 63 & 172 \\ \text { BBP } & 79 & 58 & 13 & 278 & 36 & 104 \\ \text { DEHP } & 283 & 177 & 47 & 926 & 140 & 389 \\ \text { DnOP } & 22 & 18 & 5 & 117 & 12 & 27 \\ \text { Subtotal } & \mathbf{6 2 5} & \mathbf{4 1 4} & \mathbf{1 2 3} & \mathbf{2 0 8 5} & \mathbf{3 0 2} & \mathbf{8 5 6} \\ \text { Dicarboxylic acids } & & & & & & \\ \text { Oxalic acid } & 129 & 59 & 49 & 312 & 88 & 162 \\ \text { Malonic acid } & 59 & 19 & 17 & 112 & 44 & 72 \\ \text { Sccinic acid } & 33 & 15 & 7 & 69 & 18 & 44 \\ \text { Subtotal } & \mathbf{2 2 1} & \mathbf{9 3} & \mathbf{7 3} & \mathbf{4 9 3} & \mathbf{1 5 0} & \mathbf{2 7 8} \\ \text { Anhydrosugars } & & & & & & \\ \text { Levoglucosan } & 600 & 388 & 88 & 1532 & 250 & 866 \\ \text { Galactosan } & 52 & 37 & 4 & 139 & 17 & \mathbf{9 3} \\ \text { Subtotal } & \mathbf{6 5 3} & \mathbf{4 2 5} & \mathbf{9 2} & \mathbf{1 6 7 1} & \mathbf{2 6 7} & \end{array}$

n-Fatty acids

\begin{tabular}{lcccccc} 
n-DODA & 64 & 42 & 8 & 182 & 31 & 87 \\
n-TETRADA & 63 & 31 & 17 & 149 & 40 & 86 \\
n-HEXDA & 103 & 53 & 11 & 296 & 67 & 129 \\
n-HEPTDA & 23 & 16 & 8 & 91 & 15 & 24 \\
9,12-OCTDA & 41 & 23 & 12 & 111 & 22 & 56 \\
9-OCTDA & 50 & 33 & 10 & 197 & 22 & 68 \\
n-OCTDA & 76 & 35 & 15 & 248 & 55 & 95 \\
n-EICOA & 22 & 12 & 10 & 68 & 16 & 24 \\
n-DOCOA & 36 & 22 & 9 & 108 & 19 & 53 \\
n-TETRACOA & 15 & 7 & 6 & 36 & 10 & 18 \\
n-PENTACOA & 23 & 14 & 8 & 64 & 11 & 32 \\
n-HEXACOA & 11 & 5 & 3 & 25 & 8 & 13 \\
Subtotal & $\mathbf{5 2 9}$ & $\mathbf{2 9 3}$ & $\mathbf{1 1 7}$ & $\mathbf{1 5 7 7}$ & $\mathbf{3 1 7}$ & $\mathbf{6 8 6}$ \\
\hline
\end{tabular}

Note. Organic compounds are in $\mathrm{ng} \mathrm{m}^{-3}$ while rest all other species are in $\mu \mathrm{g} \mathrm{m}^{-3}$. 
Table S2. Mass concentration and diagnostic ratio of organic species.

\begin{tabular}{|c|c|c|c|c|}
\hline Species & Pre-monsoon & Post-monsoon & Winter & Overall \\
\hline \multicolumn{5}{|l|}{ n-Alkanes } \\
\hline $\begin{array}{l}\text { Concentration } \\
\left(\mathrm{ngm}^{-3}\right)\end{array}$ & $\begin{array}{c}940 \pm 470 \\
(265-2077)\end{array}$ & $\begin{array}{c}988 \pm 578 \\
(373-2115)\end{array}$ & $\begin{array}{c}1018 \pm 505 \\
(341-2091)\end{array}$ & $\begin{array}{c}977 \pm 504 \\
(265-2115)\end{array}$ \\
\hline CPI & $\begin{array}{c}1.4 \pm 0.3 \\
(0.7-1.9)\end{array}$ & $\begin{array}{c}1.3 \pm 0.3 \\
(0.8-1.7)\end{array}$ & $\begin{array}{c}1.2 \pm 0.2 \\
(0.9-1.9)\end{array}$ & $\begin{array}{c}1.3 \pm 0.3 \\
(0.7-1.9)\end{array}$ \\
\hline LMW/HMW & $\begin{array}{c}2.1 \pm 0.9 \\
(0.6-4.6)\end{array}$ & $\begin{array}{c}1.1 \pm 0.4 \\
(0.5-1.8)\end{array}$ & $\begin{array}{l}1.5 \pm 0.6 \\
(0.7-3.5)\end{array}$ & $\begin{array}{l}1.7 \pm 0.8 \\
(0.5-4 .)\end{array}$ \\
\hline \multicolumn{5}{|l|}{ Fatty acids } \\
\hline $\begin{array}{l}\text { Concentration } \\
\left(\mathrm{ngm}^{-3}\right)\end{array}$ & $\begin{array}{c}395 \pm 98 \\
(229-584)\end{array}$ & $\begin{array}{c}479 \pm 201 \\
(202-1006)\end{array}$ & $\begin{array}{c}926 \pm 193 \\
(414-927)\end{array}$ & $\begin{array}{c}616 \pm 130 \\
(202-1006)\end{array}$ \\
\hline $\mathrm{CPI}$ & $\begin{array}{c}11.5 \pm 6.5 \\
(3.2-29.6)\end{array}$ & $\begin{array}{c}9.7 \pm 5.9 \\
(3.4-31.2)\end{array}$ & $\begin{array}{c}8.4 \pm 2.9 \\
(3.3-14.2)\end{array}$ & $\begin{array}{c}9.9 \pm 5.4 \\
(3.2-31.2)\end{array}$ \\
\hline LMW/HMW & $\begin{array}{c}11.8 \pm 8.4 \\
(3.4-42.5)\end{array}$ & $\begin{array}{c}6.1 \pm 4.0 \\
(6.1-20.9)\end{array}$ & $\begin{array}{c}8.3 \pm 5.3 \\
(3.2-30.6)\end{array}$ & $\begin{array}{c}9.3 \pm 7.0 \\
(2.3-42.5)\end{array}$ \\
\hline $\begin{array}{l}\text { Dicarboxylic acids } \\
\left(\mathrm{ngm}^{-3}\right)\end{array}$ & $\begin{array}{c}163 \pm 41 \\
(81-273)\end{array}$ & $\begin{array}{c}208 \pm 86 \\
(92-399)\end{array}$ & $\begin{array}{c}283 \pm 71 \\
(159-461)\end{array}$ & $\begin{array}{c}213 \pm 82 \\
(81-461)\end{array}$ \\
\hline $\begin{array}{l}\text { Anhydrosugars } \\
\left(\mathrm{ngm}^{-3}\right)\end{array}$ & $\begin{array}{c}302 \pm 227 \\
(88-1234)\end{array}$ & $\begin{array}{c}857 \pm 368 \\
(292-1628)\end{array}$ & $\begin{array}{c}935 \pm 323 \\
(229-1511)\end{array}$ & $\begin{array}{c}641 \pm 421 \\
(88-1628)\end{array}$ \\
\hline $\begin{array}{l}\text { Phthalates } \\
\left(\mathrm{ngm}^{-3}\right)\end{array}$ & $\begin{array}{c}335 \pm 110 \\
(168-641)\end{array}$ & $\begin{array}{c}871 \pm 240 \\
(416-1255)\end{array}$ & $\begin{array}{c}839 \pm 326 \\
(374-1659)\end{array}$ & $\begin{array}{c}626 \pm 346 \\
(168-1659)\end{array}$ \\
\hline WSOC $\left(\mu \mathrm{gm}^{-3}\right)$ & $\begin{array}{c}3 \pm 7 \\
(0.4-8.6) \\
\end{array}$ & $\begin{array}{l}13 \pm 12 \\
(2-49) \\
\end{array}$ & $\begin{array}{l}25 \pm 18 \\
(3-66)\end{array}$ & $\begin{array}{c}13 \pm 15 \\
(0.4-65.7) \\
\end{array}$ \\
\hline
\end{tabular}

NOTE. Values indicate mean \pm sd and range is within the parantheses. Pre-mons: Premonsoon (MAMJ); Post-mons: Postmonsoon (ON); Winter: DJF; Overall: all months except JAS. 
Table S3. List of Abbreviations

\begin{tabular}{llll}
\hline DEP & Diethyl Phthalate & n-TETRADA & Tetradecanoic acid \\
DBP & Dibutyl Phthalate & n-HEXDA & Hexadecanoic acid \\
BBP & Benzyl Butyl Phthalate & n-HEPTDA & Heptadecanoic acid \\
BEHP & Bis(2-Ethylhexyl) Phthalate & $9,12-$-OCTDA & 9,12-Octadecadienoic acid \\
DnOP & Di-n-Octyl Phthalate & $9-O C T D A$ & 9 (E)-Octadecenoic acid \\
OxA & Oxalic acid & n-OCTDA & Octadecanoic acid \\
MA & Malonic acid & n-EICOA & Eicosanoic acid \\
SA & Succinic acid & n-DOCOA & Docosanoic acid \\
Lev & Levoglucosan & n-TETRACOA & Tetracosanoic acid \\
Gal & Galactosan & n-PENTACOA & Pentacosanoic acid \\
n-DODA & Dodecanoic acid & n-HEXACOA & Hexacosanoic acid \\
\hline
\end{tabular}


Table S4. Neutralization factor (NF) for $\mathrm{NH}_{4}{ }^{+}, \mathrm{Ca}^{2+}, \mathrm{Mg}^{2+}$ against $\mathrm{SO}_{4}{ }^{2-}$ and $\mathrm{NO}_{3}{ }^{-}$.

\begin{tabular}{lccc}
\hline $\mathbf{N F}$ & Pre-monsoon & Post-monsoon & Winter \\
\hline $\mathrm{NH}_{4}{ }^{+}$ & $0.7 \pm 0.3$ & $1.0 \pm 0.5$ & $0.6 \pm 0.2$ \\
$\mathrm{Ca}^{2+}$ & $0.4 \pm 0.3$ & $0.4 \pm 0.3$ & $0.3 \pm 0.2$ \\
$\mathrm{Mg}^{2+}$ & $0.4 \pm 0.3$ & $0.2 \pm 0.1$ & $0.2 \pm 0.1$ \\
$\mathrm{Na}^{+}$ & $0.4 \pm 0.5$ & $0.5 \pm 0.3$ & $0.3 \pm 0.1$ \\
$\mathrm{~K}^{+}$ & $0.1 \pm 0.1$ & $0.2 \pm 0.1$ & $0.2 \pm 0.1$ \\
\hline
\end{tabular}


Table S5. Chemical and physical characteristics of different types of submicron particles.

\begin{tabular}{|l|l|l|l|}
\hline Particle classes & Particle Types & $\begin{array}{l}\text { Chemical } \\
\text { characteristics }\end{array}$ & Physical characteristics \\
\hline $\begin{array}{l}\text { Carbonaceous } \\
\text { particles }\end{array}$ & Soot & C and minor O & Chain-like aggregates. \\
\cline { 2 - 4 } & $\begin{array}{l}\text { Organics (including } \\
\text { Tar balls) }\end{array}$ & $\begin{array}{l}\text { C, O, K with coating } \\
\text { containing N and S }\end{array}$ & Spherical or irregular in shape. \\
\hline Secondary & S-rich & C, N, O, S and minor K & $\begin{array}{l}\text { No definite shape, highly } \\
\text { sensitive to electron bean, } \\
\text { mixed with organic, fine mineral } \\
\text { and metal particles. }\end{array}$ \\
\cline { 2 - 4 } & K-rich & C, N, O, S and K & $\begin{array}{l}\text { No definite shape, highly } \\
\text { sensitive to electron bean, } \\
\text { mixed with organic, fine mineral } \\
\text { and metal particles. }\end{array}$ \\
\hline Metallic particles & Metals-rich & $\begin{array}{l}\text { C, O, Ti, Mn, Fe, Zn } \\
\text { and Pb. Fe and Zn- } \\
\text { exist as Fe-O, Zn-S, } \\
\text { Zn-Pb and Zn-Mn. }\end{array}$ & $\begin{array}{l}\text { Mostly Fe-rich and Zn-rich } \\
\text { particles are spherical or } \\
\text { irregular spheroidal shape. }\end{array}$ \\
\hline Mineral particles & Fine mineral & $\begin{array}{l}\text { C, O, Si, Al, Si-Al, Fe- } \\
\text { Si, Ca, Mg, Na, K with } \\
\text { visible coatings } \\
\text { containing N and S }\end{array}$ & $\begin{array}{l}\text { Irregular shape, shines under } \\
\text { electron bean and frequently } \\
\text { coated with N and S. }\end{array}$ \\
\cline { 2 - 4 } & Fly ash & C, O, Al and Si & Spherical shape. \\
\hline
\end{tabular}


Table S6. List of selected elements, ions and molecular markers for source identification.

\begin{tabular}{ll}
\hline Sources & Molecular markers \\
\hline Industrial emissions & $\mathrm{Fe}, \mathrm{Ni}, \mathrm{Co}, \mathrm{Cd}, \mathrm{Cr}, \mathrm{DnOP}$ \\
Crustal \& road dust & $\mathrm{Ca}^{2+}, \mathrm{Mg}^{2+}, \mathrm{Na}{ }^{+}, \mathrm{Mn}, \mathrm{Pb}, \mathrm{Fe}, \mathrm{Zn}$ \\
Secondary aerosols & $\mathrm{SO}_{4}{ }^{2-}, \mathrm{NO}_{3}{ }^{-}, \mathrm{NH}_{4}^{+}, \mathrm{WSOC}, \mathrm{Oxalic}$ acid, Malonic acid, Succinic Acid \\
Vehicular emissions & $\mathrm{n}-\mathrm{C}_{17} \mathrm{H}_{36}, \mathrm{n}-\mathrm{C}_{18} \mathrm{H}_{38}, \mathrm{n}-\mathrm{C}_{19} \mathrm{H}_{40}, \mathrm{n}-\mathrm{C}_{20} \mathrm{H}_{42}, \mathrm{n}-\mathrm{C}_{21} \mathrm{H}_{44}, \mathrm{n}-\mathrm{C}_{22} \mathrm{H}_{46}, \mathrm{n}-\mathrm{C}_{23} \mathrm{H}_{48}$, \\
& $\mathrm{n}-\mathrm{C}_{24} \mathrm{H}_{50}, \mathrm{C}_{34} \mathrm{H}_{70}, \mathrm{C}_{35} \mathrm{H}_{72}, \mathrm{~Pb}, \mathrm{Cu}, \mathrm{Cr}, \mathrm{Zn}$ \\
Burning of fossil fuel & $\mathrm{n}-\mathrm{C}_{19} \mathrm{H}_{40}, \mathrm{n}-\mathrm{C}_{20} \mathrm{H}_{42}, \mathrm{n}-\mathrm{C}_{21} \mathrm{H}_{44}, \mathrm{n}-\mathrm{C}_{22} \mathrm{H}_{46}, \mathrm{n}-\mathrm{C}_{23} \mathrm{H}_{48}, \mathrm{n}-\mathrm{C}_{24} \mathrm{H}_{50}, \mathrm{n}-\mathrm{C}_{25} \mathrm{H}_{52}$, \\
& $\mathrm{n}-\mathrm{C}_{26} \mathrm{H}_{54}, \mathrm{n}-\mathrm{C}_{27} \mathrm{H}_{56}, \mathrm{n}-\mathrm{C}_{28} \mathrm{H}_{58}, \mathrm{n}-\mathrm{C}_{29} \mathrm{H}_{60}, \mathrm{n}-\mathrm{C}_{30} \mathrm{H}_{62}, \mathrm{n}-\mathrm{C}_{31} \mathrm{H}_{64}$ \\
Biomass and waste/refuse & Levoglucosan, Galactosan, Zn, K+, Cl, DEP, DBP, BBP, DEHP, D-n- \\
burning & OP, n-DODA, n-TETRADA, n- $\mathrm{C}_{29} \mathrm{H}_{60}, \mathrm{n}-\mathrm{C}_{31} \mathrm{H}_{64}$ \\
& $\mathrm{n}-\mathrm{TETRADA}, \mathrm{n}-\mathrm{HEXDA}, 9,12-\mathrm{OCTDA}, 9-\mathrm{OCTDA}, \mathrm{n}-\mathrm{OCTDA}, \mathrm{n}-\mathrm{C}_{29} \mathrm{H}_{60}$, \\
Biogenic \& residential emissions & $\mathrm{n}-\mathrm{C}_{31} \mathrm{H}_{64}, \mathrm{n}-\mathrm{C}_{33} \mathrm{H}_{68}$ \\
\hline
\end{tabular}



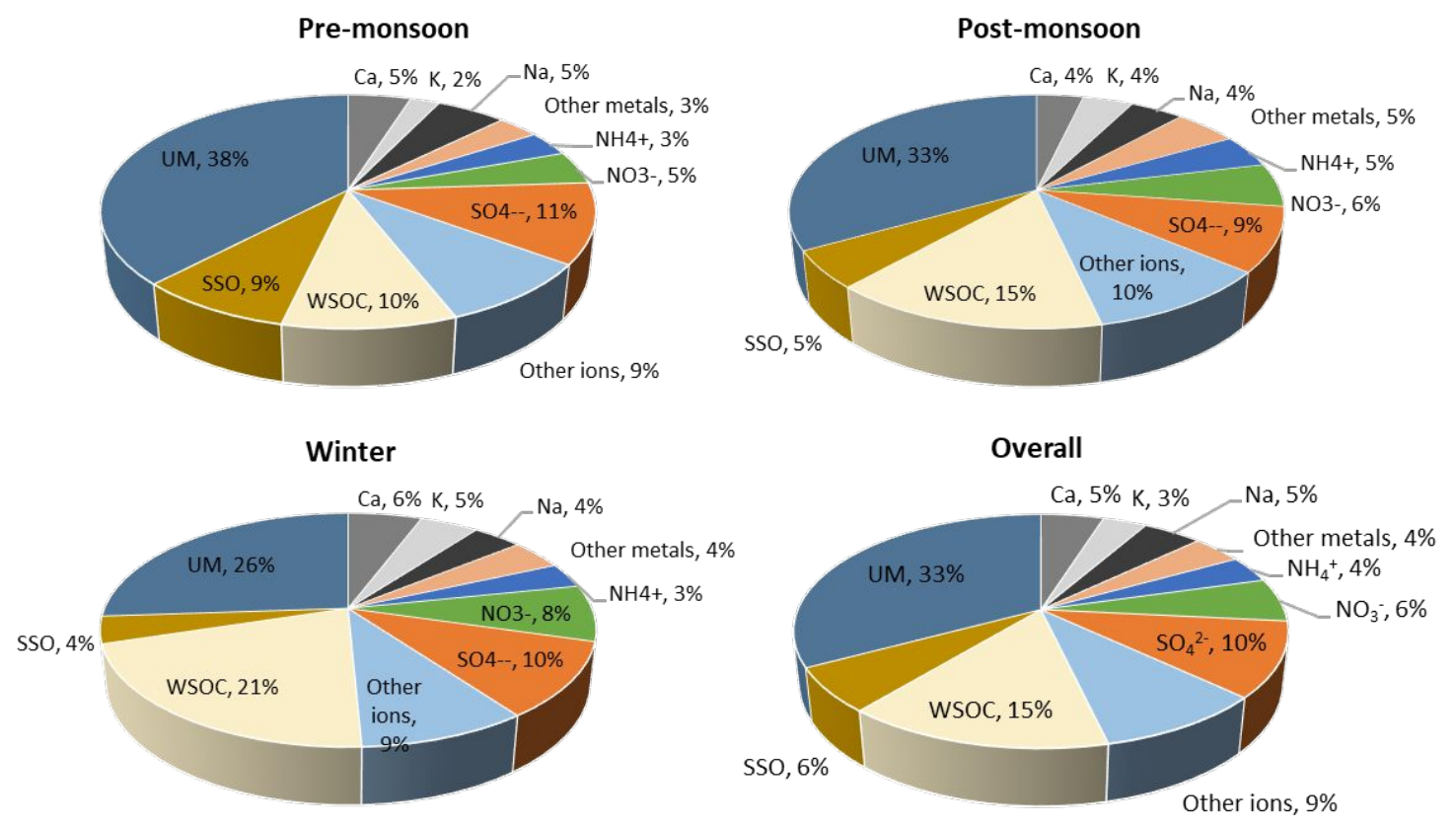

Fig. S1. Seasonal variation in relative contribution of various species in submicron particulates. 


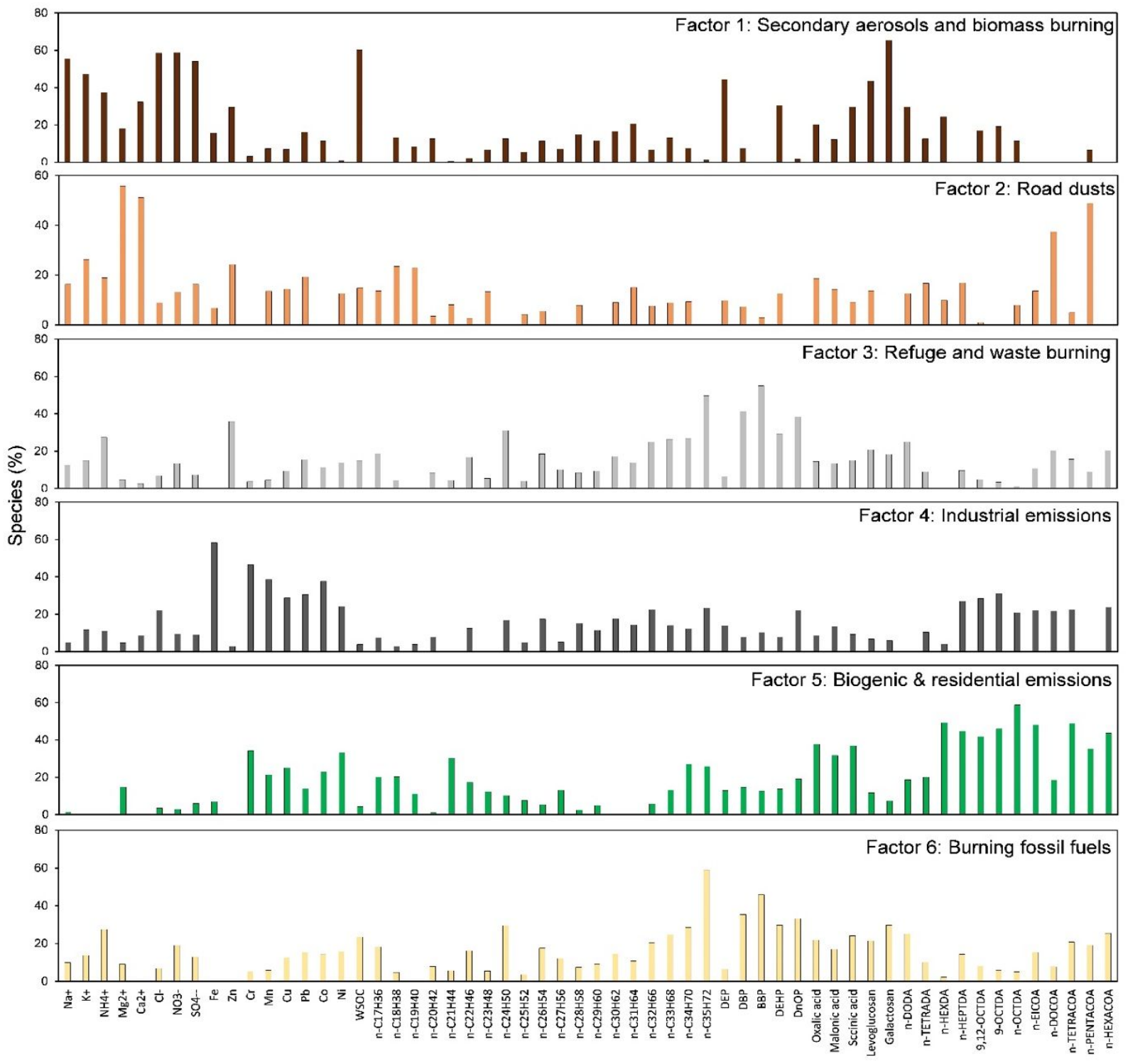

Fig. S2. Factor profile for various sources. 


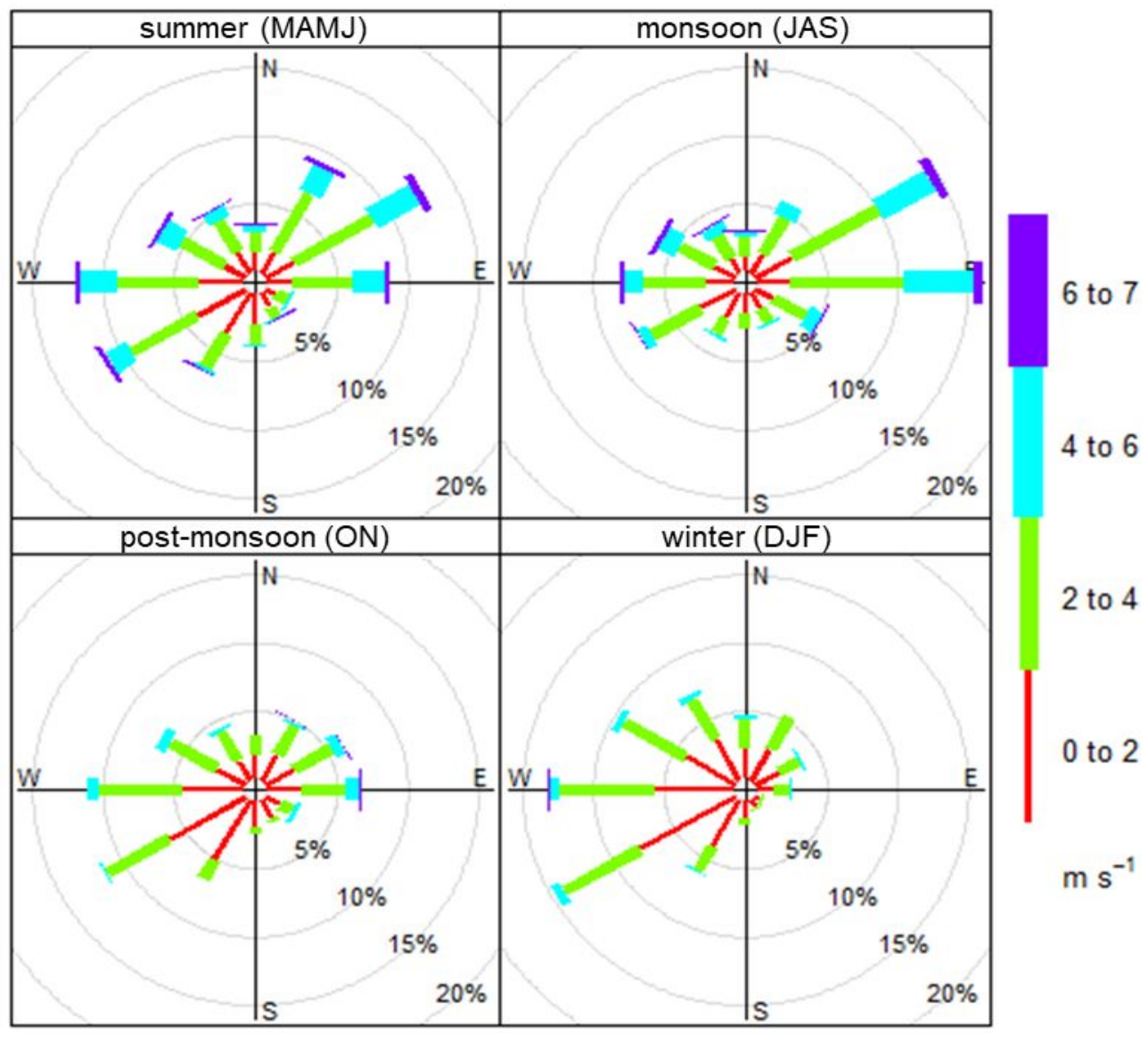

Fig. S3. Wind vectors over the study site. 